\title{
Universal early-time response in high-contrast electromagnetic scattering
}

\author{
Peter B. Weichman \\ ${ }^{2}$ ALPHATECH, Inc., 6 New England Executive Place, Burlington, MA 01803
}

(Dated: November 2, 2018)

\begin{abstract}
The time-domain response of highly conducting targets following a rapidly terminated electromagnetic pulse displays three distinct regimes: early, intermediate and late time. The intermediate and late times are characterized by a superposition of exponentially decaying eigenmodes. At early time an ever increasing number of rapidly decaying modes contribute, with the result that the scattered electric field displays a universal $t^{-1 / 2}$ power law which emerges from the diffusive decay of a pattern of surface currents induced by the pulse. The power law amplitude reflects the surface geometry of the target, a property that may prove useful in buried target classification in geophysical remote sensing applications.
\end{abstract}

PACS numbers: 03.50.De, 41.20.-q, 41.20.Jb

Remote detection and classification of buried targets is a key goal in a number of important environmental geophysical applications, such as toxic waste drum, and landmine and unexploded ordnance (UXO) remediation [1]. A common tool used for detection of highly conducting metallic targets is the time-domain electromagnetic (TDEM) method, in which an inductive coil is used to transmit EM pulses into the ground. Following each pulse, the voltage $V(t)$ induced by the scattered field is detected by a receiver coil [2]. The magnitude and lifetime of the currents induced in the target, and hence $V(t)$, increase with its size and conductivity. Standard TDEM sensors are capable of resolving anomalies from very small (of order 1 gram) metal targets [3] , and are therefore well suited to detection of relatively large buried conducting bodies such as UXO. However, since TDEM is a very low frequency (typically of order $100 \mathrm{~Hz}$ ) method, its spatial resolution (limited by the target depth and the sensor diameter) is also very low [4]. Therefore, the raw signal amplitude and lifetime provide gross measures of the target size and conductivity, but give no direct information about its geometry and other physical characteristics that would enable discrimination between, say, UXO and similarly sized clutter.

Lacking direct target geometry signatures in TDEM data (analogous to, e.g., optical and radar images of unoccluded targets), one seeks indirect measures via more detailed analysis of $V(t)$. This signal contains information about both intrinsic (target size, shape, geometry, and other physical characteristics) and extrinsic (relative target-sensor orientation, transmitter and receiver coil geometries, pulse waveform, etc.) properties, and the key to discrimination is the extraction of the former from the "background" of the latter. We shall show that such an analysis divides naturally into early, intermediate and late time domains. Intermediate time is characterized by a finite superposition of exponential decays, the slowest of which eventually dominates and defines late time. Early time is characterized by an essentially infinite number of exponential decays which superimpose to generate a $1 / \sqrt{t}$ universal power law divergence in $V(t)$ [5]. The importance of this latter interval is greatly en- hanced for ferrous targets whose response is so slow that it may in fact comprise the full measured range of $V(t)$.

To focus the discussion consider the following model. At low frequencies the dielectric function in the ground and in the target is dominated by the its imaginary part, $\epsilon=4 \pi i \sigma / \omega[\underline{6}]$, where $\sigma(\mathbf{x})$ is the dc conductivity (in Gaussian units), and the Maxwell equations may be reduced to a single equation for the vector potential,

$$
\nabla \times\left(\frac{1}{\mu} \nabla \times \mathbf{A}\right)+\frac{4 \pi \sigma}{c^{2}} \partial_{t} \mathbf{A}=\frac{4 \pi}{c} \mathbf{j}_{S},
$$

with magnetic induction $\mathbf{B}=\nabla \times \mathbf{A}$ and gauge chosen so that the electric field is $\mathbf{E}=-(1 / c) \partial_{t} \mathbf{A}$. The transmitter loop is modelled by the source current density $\mathbf{j}_{S}(\mathbf{x}, t)=I_{0}(t) \mathbf{C}_{T}(\mathbf{x})$, where the current $I_{0}$ consists of a periodic sequence of rapidly terminated pulses, and $\mathbf{C}_{T}(\mathbf{x})$ defines the transmitter loop. The magnetic field is $\mathbf{H}=\mathbf{B} / \mu$, where $\mu(\mathbf{x})$ is the (relative) permeability. The conductivity and permeability are separated into background $\left(\sigma_{b}(\mathbf{x}), \mu_{b}(\mathbf{x})\right)$ and conducting target $\left(\sigma_{c}(\mathbf{x})\right.$, $\left.\mu_{c}(\mathbf{x})\right)$ components, where $\sigma_{c}, \mu_{c}$ vanish outside the target volume $V_{c}$, and it is assumed only that $\sigma_{b} / \sigma_{c} \ll 1$.

Equation (11) is a vector diffusion equation with diffusion constant $D=c^{2} / 4 \pi \mu \sigma$. Typical values are $D_{b}=$ $8.0 \times 10^{10} \mathrm{~m}^{2} / \mathrm{s}$ for a nonmagnetic background with resistivity of $10 \Omega \mathrm{m} ; D_{c}=2.3 \times 10^{2} \mathrm{~m}^{2} / \mathrm{s}$ for an aluminum target with resistivity $2.8 \times 10^{-8} \Omega \mathrm{m} ;$ and $D_{c}=4.0 \mathrm{~m}^{2} / \mathrm{s}$ for a steel target with relative permeability 200 and resistivity $8.9 \times 10^{-8} \Omega \mathrm{m}$. EM signal propagation distance $d$ in time $t$ may be estimated via $d \sim \sqrt{D t}$. Early-time results will require that $\tau_{b}=R^{2} / D_{b} \ll \tau_{c}=L_{c}^{2} / D_{c}$, where $R$ is the distance between the sensor and the target, and $L_{c}$ is the latter's linear size: target-sensor propagation time should be instantaneous on the time scale of the electrodynamics of the target itself [7]. The associated condition $R \ll L_{c} \sqrt{D_{b} / D_{c}}$ is easily satisfied for centimeter scale targets at tens of meters depth, and is even less stringent for larger targets. The off-ramp time, $\tau_{r}$, for the transmitted pulse is assumed to satisfy $\tau_{r} \ll \tau_{c}$, so that pulse termination also occurs essentially instantaneously on the scale of the target dynamics (no particular relation between $\tau_{r}$ and $\tau_{b}$ is required). 
In order to further elucidate the various time scales in the problem, consider the homogeneous version of (1), valid between pulses. The general solution takes the form of a superposition of exponentially decaying eigenmodes

$$
\mathbf{A}(\mathbf{x}, t)=\sum_{n=1}^{\infty} A_{n} \mathbf{a}^{(n)}(\mathbf{x}) e^{-\lambda_{n}\left(t-t_{0}\right)}
$$

in which $t_{0}$ marks the beginning of the free decay window, and the mode shapes $\mathbf{a}^{(n)}$ and decay rates $\lambda_{n}$ satisfy the eigenvalue equation

$$
\nabla \times\left(\frac{1}{\mu} \nabla \times \mathbf{a}^{(n)}\right)=\frac{4 \pi \sigma \lambda_{n}}{c^{2}} \mathbf{a}^{(n)} .
$$

These modes (which may be orthonormalized by noting that $\sqrt{\sigma} \mathbf{a}^{(n)}$ are eigenfunctions of a self adjoint operator) correspond to special current density patterns $\mathbf{j}^{(n)}(\mathbf{x})=$ $\left(\lambda_{n} / c\right) \sigma(\mathbf{x}) \mathbf{a}^{(n)}(\mathbf{x})$ with decaying amplitude, but timeindependent spatial structure [8]. The spectrum $\left\{\lambda_{n}\right\}$ is bounded below, with fundamental decay rate $\lambda_{1} \sim 1 / \tau_{c}$ governed by the target size, but unbounded from above [9, 10, 11], with more rapidly decaying modes having spatial structure on ever smaller scales. Since $\sigma_{b} \ll \sigma_{c}$ currents in the background are negligible compared to those in the target, and it follows that $\lambda_{n}$, as well as the internal structure of $\mathbf{a}^{(n)}(\mathbf{x}), \mathbf{x} \in V_{c}$, are essentially independent of the background [12]. In this sense $\lambda_{n}$ and $\mathbf{a}^{(n)}$ are intrinsic properties of the target. Explicitly, one finds

$$
\begin{aligned}
A_{n} & =\frac{4 \pi}{c^{2}} I_{n} \int_{C_{T}} \mathbf{a}^{(n) *}(\mathbf{x}) \cdot d \mathbf{l} \\
I_{n} & =\int_{-\infty}^{t_{0}} d t^{\prime} I_{0}\left(t^{\prime}\right) e^{-\lambda_{n}\left(t_{0}-t^{\prime}\right)},
\end{aligned}
$$

where the transmitter coil here is an idealized 1D curve $C_{T}$. If the coil has $N_{T}$ windings then $I_{0}(t)=N_{T} i_{0}(T)$, where $i_{0}$ is the actual current. The voltage measured in the receiver loop is then

$$
\begin{aligned}
V(t) & =\sum_{n=1}^{\infty} V_{n} e^{-\lambda_{n}\left(t-t_{0}\right)} \\
V_{n} & =\frac{\lambda_{n} N_{R}}{c} A_{n} \int_{C_{R}} \mathbf{a}^{(n)}(\mathbf{x}) \cdot d \mathbf{l},
\end{aligned}
$$

in which $C_{R}$ is the idealized $1 \mathrm{D}$ receiver loop and $N_{R}$ is the number of windings.

The excitation coefficients $A_{n}$ and $V_{n}$ depend on both intrinsic (eigenmode) and extrinsic (transmitter/receiver loop geometry, position, orientation, etc.) information. Given only $V(t)$, absent any information regarding the measurement geometry, target classification relies entirely on the extractable subset of decay rates $\lambda_{n}$. The mathematical problem is equivalent to the famous "Can you hear the shape of a drum?" (i.e., to what extent is the shape of a struck drumhead determined by its frequency spectrum?), but is practically much more difficult because no analogue of the Fourier transform exists for directly estimating the $\lambda_{n}$. In contrast, if detailed measurement information is available, direct prediction of the amplitudes, and hence of the full signal $V(t)$ is possible. A classification scheme may then be developed based on a search for the target model that directly minimizes the difference between the measured and predicted data [1], thus circumventing the (generally unstable) problem of direct estimation of $\left\{\lambda_{n}\right\}$ from noisy data.

The number of substantially excited modes in (2) depends on $\tau_{r}$ (50-100 $\mu$ s in many commercial systems). Roughly, the terminating pulse will excite a subset (depending on the extrinsic parameters) of those modes with $\lambda_{n} \lesssim \lambda_{r} \equiv 1 / \tau_{r}$. The higher order modes will decay very rapidly, but still contribute strongly at early time $t-t_{0}=\mathcal{O}\left(\tau_{r}\right)$. Realistically, one can hope to accurately compute only the first few hundred modes 10, 11]. If the largest computable decay rate $\lambda_{\max }$ is smaller than $\lambda_{r}$, then the interval $0 \leq t-t_{0} \leq 1 / \lambda_{\max }$ will not be accurately modelled. For ferrous targets it is often the case that $1 / \lambda_{1}$ exceeds the measurement window and the response is entirely early time. The remainder of this paper is therefore concerned with the development of a complementary theory that deals with this interval. By combining this theory with the mode analysis, a comprehensive model of the entire time-domain signal emerges.

The analysis proceeds in three steps. (1) An "initial condition" for the free dynamics, consisting of a pattern of currents confined to the surface of the target, is computed. (2) The time-development of this surface current, namely its diffusion into the interior of the target, is computed. (3) Finally, this solution is used to compute the external field generated at the sensor.

Step 1: The rapid quenching of the transmitter current leads to an outgoing EM pulse that scatters off the target in a complicated way, but exits the target region by some transient time $t_{\text {tr }}=t_{0}+\tau_{\text {tr }}$, with $\tau_{\text {tr }}=\mathcal{O}\left(\tau_{b}\right)$. The assumption $\tau_{b} \ll \tau_{c}$ implies that the internal field $\mathbf{A}\left(\mathbf{x}, t_{0}-\tau_{r}\right)$ just prior to the pulse termination, remains essentially fixed during the interval $-\tau_{r}<t-t_{0}<\tau_{\text {tr }}$, responding only in a thin shell near the boundary, $\partial V_{c}$. More precisely, at high frequencies where the target skin depth $\delta_{c}=\sqrt{2 D_{c} / \omega}$ is much smaller than the scale of tangential variation of $\mathbf{A}$, the internal field near the surface, with local normal $\hat{\mathbf{n}}$, takes the form 13

$$
\mathbf{A}(\mathbf{x}, \omega)=\mathbf{A}^{\|}(\mathbf{r}, \omega) e^{-|z| \sqrt{-i \omega / D_{c}}},
$$

in which $z$ the coordinate along $\hat{\mathbf{n}}$, and $\mathbf{r}$ is orthogonal to it, and $\hat{\mathbf{n}} \cdot \mathbf{A} \|=0$. Continuity of $\hat{\mathbf{n}} \times \mathbf{A}$ implies that $\mathbf{A}^{\|}$ is also the tangential component of the external field. In the time domain, (6) becomes

$$
\begin{aligned}
\mathbf{A}(\mathbf{x}, t) & =\mathbf{A}_{0}(\mathbf{x}, t)+\Delta \mathbf{A}(\mathbf{x}, t) \\
\Delta \mathbf{A}(\mathbf{x}, t) & =\int_{t_{0}-\tau_{r}}^{t} d t^{\prime} \mathbf{A}^{\|}\left(\mathbf{r}, t^{\prime}\right) \frac{|z|}{t-t^{\prime}} \frac{e^{-z^{2} / 4 D_{c}\left(t-t^{\prime}\right)}}{\sqrt{4 \pi D_{c}\left(t-t^{\prime}\right)}}
\end{aligned}
$$

valid for $t-t_{0} \ll \tau_{c}$, demonstrating the diffusion of the signal inwards from the surface. The current density is 
given by the same expression, but with $\mathbf{A}^{\|}$replaced by $\sigma_{c} \mathbf{E}^{\|} \equiv-\left(\sigma_{c} / c\right) \partial_{t} \mathbf{A}^{\|}$. Integrating over $z$, at $t_{\mathrm{tr}}$ there is an effective surface current,

$$
\mathbf{K}\left(\mathbf{r}, t_{\mathrm{tr}}\right)=\int_{t_{0}-\tau_{r}}^{t_{0}+\tau_{\text {tr }}} \frac{d t^{\prime}}{2 \pi} \sqrt{\frac{\sigma_{c}}{\mu_{c}\left(t-t^{\prime}\right)}} \partial_{t}^{\prime} \mathbf{A}^{\|}\left(\mathbf{r}, t^{\prime}\right)
$$

confined to a thin shell with width $\sqrt{D_{c}\left(\tau_{r}+\tau_{\mathrm{tr}}\right)}$.

Equation (8) provides a rigorous foundation for $\mathbf{K}$, expressing it in terms the external field at the boundary, but the latter has no simple form and is generally unknown. We now describe an alternate procedure for its direct computation via a self-consistency argument. At time $t_{\text {tr }}$ (11) is to be solved with $\mathbf{j}_{S}=0$. Since all background transients have died out, the $\sigma \partial_{t} \mathbf{A}$ term is of relative order $D_{c} R^{2} / D_{b} L_{c}^{2}$ compared to the curl term and may be dropped. It follows that $\mathbf{H}=\mu_{b}^{-1} \nabla \times \mathbf{A}=-\nabla \Phi$ is the gradient of a magnetic potential satisfying

$$
\nabla \cdot\left(\mu_{b} \nabla \Phi\right)=0 .
$$

The solution $\Phi_{0}$ to this equation must satisfy appropriate boundary conditions on $\partial V_{t}$, namely $\hat{\mathbf{n}} \cdot\left(\mu_{b} \mathbf{H}_{b}-\mu_{c} \mathbf{H}_{c}\right)=$ 0 and $\hat{\mathbf{n}} \times\left(\mathbf{H}_{b}-\mathbf{H}_{c}\right)=(4 \pi / c) \mathbf{K}[13$. In both cases, $\mathbf{H}_{c}=\nabla \times \mathbf{A}_{c}$ is obtained from the initial internal field $\mathbf{A}\left(\mathbf{x}, t_{0}-\tau_{r}\right)$ evaluated at the boundary. The first condition imposes a unique solution on $\Phi$ via the Neumann boundary condition

$$
-\hat{\mathbf{n}} \cdot \nabla \Phi_{0}=\frac{\mu_{c}}{\mu_{b}} \hat{\mathbf{n}} \cdot \mathbf{H}_{c}\left(\mathbf{r}, z=0^{-}\right),
$$

with formal solution

$$
\Phi_{0}(\mathbf{x})=\int_{\partial V_{c}} d^{2} r^{\prime} g_{N}\left(\mathbf{x}, \mathbf{r}^{\prime}\right) \frac{\mu_{c}}{\mu_{b}} \hat{\mathbf{n}} \cdot \mathbf{H}_{c}\left(\mathbf{r}^{\prime}\right),
$$

where $g_{N}$ is the Neumann green function satisfying $-\nabla$. $\left(\mu_{b} \nabla g_{N}\right)=\delta\left(\mathbf{x}-\mathbf{x}^{\prime}\right)$ with boundary condition $\hat{\mathbf{n}} \cdot \nabla g_{N}=$ 0 . The second condition determines $\mathbf{K}$ :

$$
\mathbf{K}=-\frac{c}{4 \pi} \hat{\mathbf{n}} \times\left(\nabla \Phi_{0}+\mathbf{H}_{c}\right) .
$$

Step 2: In order to investigate the subsequent evolution of the surface current $\mathbf{K}$ we take advantage of the rapid variation of the fields near the surface with $z$. Thus, the $z$-derivatives dominate (11), and to leading order in the small parameter $\epsilon=\sqrt{D_{c}\left(t-t_{\mathrm{tr}}\right) / L_{c}^{2}}$ one need only solve the one-dimensional diffusion equation

$$
D_{c} \partial_{z}^{2} \mathbf{E}^{\perp}+\partial_{t} \mathbf{E}=0, z<0,
$$

with initial condition $\mathbf{E}\left(t_{\mathrm{tr}}\right)=\sigma_{c}^{-1} \mathbf{K} \delta(z)$. Here $\mathbf{E}^{\perp}=$ $\mathbf{E}-\hat{\mathbf{n}}(\hat{\mathbf{n}} \cdot \mathbf{E})$ is the tangential part of $\mathbf{E}$, and $\mu, \sigma, D$ are treated as constants on either side of the boundary. Since the external field varies only on the scales $L_{c}, R$, to leading order one has $\partial_{z} \mathbf{E}^{\perp}\left(z=0^{+}\right)=0$. Continuity of $\mathbf{E}^{\perp}$ therefore imposes the Neumann boundary condition $\partial_{z} \mathbf{E}^{\perp}\left(z=0^{-}\right)=0$. The solution to (13) is therefore

$$
\mathbf{E}(\mathbf{x}, t)=\mathbf{E}_{0}(\mathbf{x}, t)+\frac{2}{\sigma_{c}} \frac{e^{-z^{2} / 4 D_{c}\left(t-t_{\mathrm{tr}}\right)}}{\sqrt{4 \pi D_{c}\left(t-t_{\mathrm{tr}}\right)}}, z<0,
$$

corresponding to a diffusive Gaussian spread with rapid $z$-dependence is on the scale $\sqrt{D_{c}\left(t-t_{\text {tr }}\right)}=\epsilon L_{c}$. By integrating with respect to time, and enforcing the condition that $\mathbf{A}$ should approach the background solution $\mathbf{A}_{0}$ for large $z / \epsilon L_{c}$, one obtains

$$
\begin{array}{r}
\Delta \mathbf{A}(\mathbf{x}, t)=\frac{4 \pi \mu_{c}}{c} \mathbf{K}(\mathbf{r})\left[4 D_{c}\left(t-t_{\mathrm{tr}}\right) \frac{e^{-z^{2} / 4 D_{c}\left(t-t_{\mathrm{tr}}\right)}}{\sqrt{4 \pi D_{c}\left(t-t_{\mathrm{tr}}\right)}}\right. \\
\left.-|z| \operatorname{erfc}\left(\frac{|z|}{\sqrt{4 D_{c}\left(t-t_{\mathrm{tr})}\right)}}\right)\right]
\end{array}
$$

where $\operatorname{erfc}(z)=(2 / \sqrt{\pi}) \int_{z}^{\infty} e^{-s^{2}} d s$ is the complementary error function. Since $\mathbf{K}$ is, in fact, spread over a width $\sqrt{D_{c} \tau_{\text {tr }}}$, equations (14) and (15) are accurate only in the range $\tau_{\text {tr }} \ll t-t_{0} \ll \tau_{c}$ where the precise microscopic structure (17) has been washed out by the diffusion kernel.

Step 3: Equation (14) evaluated at $z=0^{-}$provides the necessary boundary condition for evaluation of the external field to leading order in $\epsilon$. Note that $\mathbf{E}\left(z=0^{-}\right) \approx \mathbf{K} \sqrt{4 \mu_{c} / \sigma_{c} c^{2}\left(t-t_{\mathrm{tr}}\right)}$ diverges, and continuity of $\mathbf{E}^{\perp}$ leads one to expect a corresponding divergence in the external electric field. We exhibit this formally through a correction $\Delta \Phi$ to the magnetic potential. Thus, the normal component of the curl of (15) leads to the boundary value $\hat{\mathbf{n}} \cdot \mathbf{B}\left(z=0^{-}\right)=\hat{\mathbf{n}} \cdot\left(\mathbf{B}_{0}+\Delta \mathbf{B}\right)$, where

$$
\hat{\mathbf{n}} \cdot \Delta \mathbf{B}=4 \sqrt{t-t_{\mathrm{tr}}} \hat{\mathbf{n}} \cdot \nabla \times\left(\sqrt{\mu_{c} / \sigma_{c}} \mathbf{K}\right) .
$$

involves only derivatives with respect to the tangential coordinate $\mathbf{r}$, and is valid even if $\mu_{c}, \sigma_{c}$ vary on the scale $L_{c}$. We therefore obtain $\Phi=\Phi_{0}+\Delta \Phi$, with boundary condition $-\mu_{b} \hat{\mathbf{n}} \cdot \Delta \Phi=\hat{\mathbf{n}} \cdot \Delta \mathbf{B}$, and hence to $\mathcal{O}(\epsilon)$,

$$
\Delta \Phi(\mathbf{x})=\int d^{2} r^{\prime} g_{N}\left(\mathbf{x}, \mathbf{r}^{\prime}\right) \frac{1}{\mu_{b}} \hat{\mathbf{n}} \cdot \Delta \mathbf{B}\left(\mathbf{r}^{\prime}\right),
$$

which is proportional to $\sqrt{t-t_{\mathrm{tr}}}$. The correction to the external vector potential is obtained by solving the auxiliary pair of equations

$$
\begin{aligned}
\nabla \times \Delta \mathbf{A} & =-\mu_{b} \nabla \Delta \Phi \\
\nabla \cdot\left(\sigma_{b} \Delta \mathbf{A}\right) & =0,
\end{aligned}
$$

and is clearly also proportional to $\sqrt{t-t_{\mathrm{tr}}}$. The electric field correction $\Delta \mathbf{E}=-(1 / c) \partial_{t} \Delta \mathbf{A} \propto\left(t-t_{\mathrm{tr}}\right)^{-1 / 2}$ therefore has the promised square root early time divergence. Measurements of magnetic field or voltage (via the time derivative of the of the integral of the magnetic flux through receiver loop area) follow directly from (17).

We end by illustrating the early time behavior using exact analytical results for a homogeneous sphere of radius $a$ in a homogeneous background [14]. We consider also an initial static transmitted field, so that the initial magnetic field is everywhere described by a scalar potential. The initial solution is a superposition of spherical harmonics,

$$
\Phi_{\text {init }}^{l m}=Y_{l m} \begin{cases}(r / a)^{l}, & r<a \\ b_{\text {init }}^{l m}(r / a)^{l}+c_{\text {init }}^{l m}(a / r)^{l+1}, & r>a .\end{cases}
$$


with $c_{\text {init }}^{l m}=1-b_{\text {init }}^{l m}=\left(1-\mu_{c} / \mu_{b}\right) \frac{l}{2 l+1}(l=1$ corresponds to the standard case of a uniform illumination field, leading to a dipole response). At $t_{\mathrm{tr}}$ the $r<a$ solution remains the same, while $b_{0}^{l m}$ vanishes. The boundary condition (10) then leads to $\Phi_{0}^{l m}=c_{0}^{l m} Y_{l m}$, with $c_{0}^{l m}=-l \mu_{c} /(l+1) \mu_{b}$. From (12 surface current is,

$$
\mathbf{K}^{l m}=\frac{i c}{4 \pi a}\left(1+\frac{l}{l+1} \frac{\mu_{c}}{\mu_{b}}\right) \sqrt{l(l+1)} \mathbf{X}_{l m} .
$$

From (16) and (17) one then obtains,

$$
\begin{aligned}
\Delta \Phi^{l m} & =-\phi_{l}(t)\left(\frac{a}{r}\right)^{l+1} Y_{l m} \\
\phi_{l}(t) & \equiv \frac{\mu_{c} l}{\mu_{b} a}\left(1+\frac{l}{l+1} \frac{\mu_{c}}{\mu_{b}}\right) \sqrt{\frac{4 D_{c}\left(t-t_{\mathrm{r}}\right)}{\pi}}
\end{aligned}
$$

and the external fields are given by

$$
\Delta \mathbf{B}^{l m}=i \mu_{b} \sqrt{\frac{l+1}{l}} \phi_{l}(t) \nabla \times\left[\left(\frac{a}{r}\right)^{l+1} \mathbf{X}_{l m}\right]
$$

$$
\Delta \mathbf{A}^{l m}=i \mu_{b} \sqrt{\frac{l+1}{l}} \phi_{l}(t)\left(\frac{a}{r}\right)^{l+1} \mathbf{X}_{l m}
$$

which each display $\sqrt{t}$ cusps, while

$$
\Delta \mathbf{E}^{l m}=-\frac{i \mu_{b}}{c} \sqrt{\frac{l+1}{l}} \frac{\phi_{l}(t)}{2\left(t-t_{\mathrm{tr}}\right)}\left(\frac{a}{r}\right)^{l+1} \mathbf{X}_{l m}
$$

has the $1 / \sqrt{t}$ divergence. Here $\mathbf{X}_{l m}=-i[l(l+l)]^{-1 / 2} \mathbf{x} \times$ $\nabla Y_{l m}$ are the vector spherical harmonics [13]. The spatial decay rate of the signal increases with $l$, but all harmonics have the same universal power law time-dependence.

The author is indebted to E. M. Lavely for numerous discussions. The support of SERDP, through contract No. DACA 72-02-C-0029, is gratefully acknowledged.
[1] See, e.g., http://www.serdp.org/research/research.html for a list of ongoing projects in these areas.

[2] Solid state magnetoresistive sensors (presently used, e.g., in recording heads in magnetic data strorage devices) are also being developed, yielding direct millimeter-scale vector measurements of the magnetic field $\mathbf{B}(t)$, but will not effectively compete with standard meter-scale induction measurements without greater noise reduction. See, e.g., R. J. Wold, P. B. Weichman, M. Tondra, D. Reed, E. Lange and A. Becker, "Proof-of-concept of a standoff UXO detection system using SDT sensor arrays," Proc. UXO/Countermine Forum (April 2001).

[3] See, e.g., C. V. Nelson and T. B. Huynh, "Wide bandwidth time decay responses from low metal mines and ground voids," Proc. SPIE AeroSense Symp. Detect. Tech. for Mines and Minelike Targets VI (April 2001).

[4] Low frequency is crucial for reasonable $(>10 \mathrm{~m})$ depth sensitivity. At ground penetrating radar frequencies, where the short wavelength $(<10 \mathrm{~cm})$ ensures good spatial resolution, the EM skin depth is at best a few meters, and much smaller still in wet soil. In addition, the dielectric contrast between the ground and the target (and similarly sized clutter, such as rocks) is greatly reduced, thus also reducing target classification capability.

[5] In an analogous effect, molecular diffusion produces an initial linear decay of the NMR signal from fluids in porous media. The coefficient reflects the surface-tovolume ratio of the pores. See P. P. Mitra, P. N. Sen, L. M. Schwartz and P. LeDoussal, Phys. Rev. Lett. 68, 3555 (1992).

[6] This actually need only be true inside the target. The background may be insulating $\left(\epsilon=\epsilon^{\prime}\right.$ real), with no significant change in the results. It is required only that signals, be they diffusive or wavelike, propagate much faster in the background than in the target.

[7] In an insulating background one has instead $d \sim c t / \sqrt{\epsilon_{b}^{\prime}}$, and the much less stringent condition $R / c \ll \tau_{c}$.

[8] The spectrum of target modes $\left\{\lambda_{n}\right\}$ is actually embedded in the continuum of background decay modes. E.g., for an infinite homogeneous medium, the modes are plane waves $\mathbf{a}(\mathbf{x} ; \mathbf{q})=\mathbf{a}_{0} e^{i \mathbf{q} \cdot \mathbf{x}}$, with $\mathbf{a}_{0} \cdot \mathbf{q}=0$ and decay rate $\lambda(\mathbf{q})=$ $D_{b} \mathbf{q}^{2}$. This background response is used in geophysical surveys to estimate the ground conductivity, but must be subtracted out (e.g., using data taken far from the target) to isolate the target response.

[9] Analytical solutions are possible only for spherical targets. Recently developed numerical techniques [the general theory is developed in P. B. Weichman, "Mean field approach to high contrast scattering" (preprint, 2003)] for solving (3) now produce spectra for oblate and prolate spheroidal targets with a broad range of aspect ratios: see Refs. 10, 11] below.

[10] P. B. Weichman, "Rapid computation of time-domain response of metallic scatterers for real-time discrimination," Proc. 2001 SAGEEP meeting (March 2001).

[11] P. B. Weichman and E. M. Lavely, "Study of inverse problems for buried UXO discrimination based on EMI sensor data," Proc. SPIE AeroSense Symp. Detect. Tech. for Mines and Minelike Targets VIII (April 2003).

[12] The external field, however, will be distorted by background variations, but there is negligible feedback on the internal field. This may be formalized via the Green function integral formulation of (3) which reduces the eigenvalue equation to one for the internal field alone.

[13] See, e.g., J. D. Jackson, Classical Electrodynamics (John Wiley and Sons, New York, 1975).

[14] Further examples for ellipsoidal targets, where partial analytic results may be obtained, along with more extensive calculations of finite corrections to the leading $1 / \sqrt{t}$ behavior at higher order in $\epsilon$, will be presented elsewhere: P. B. Weichman, manuscript in preparation. 